\title{
PATA TYPE BEST PROXIMITY POINT RESULTS IN METRIC SPACES
}

\author{
N. SALEEM, M. ABBAS, B. BIN-MOHSIN, AND S. RADENOVIĆ
}

Received 29 November, 2018

\begin{abstract}
The aim of this paper is to initiate the study of best proximity point and optimal coincidence point results of some $\alpha$-Pata-proximal admissible contraction of type-I and type-II in the framework of complete metric space. Some examples are presented to support the results obtained herein. Our results unify, extend and generalize various existing results in literature.
\end{abstract}

2010 Mathematics Subject Classification: 47H10; 47H04; 47H07

Keywords: metric space, $\alpha$-Pata-proximal admissible contraction of type-I and type-II, generalized $\alpha$-Pata-proximal contraction, optimal coincidence best proximity point

\section{INTRODUCTION AND PRELIMINARIES}

Banach contraction principle states that if $T$ is a self mapping on a complete metric space $(X, d)$, then $T$ has a unique fixed point in $X$ provided that for all $x, y \in X$, there exists $k \in[0,1)$ such that the following condition hold:

$$
d(T x, T y) \leq k d(x, y) .
$$

A self mapping $T$ satisfying the above inequality is said to be a contraction mapping.

A self mapping $T$ on $(X, d)$ is called Kannan contraction if for all $x, y \in X$, there exists $\gamma \in[0,1)$ such that

$$
d(T x, T y) \leq \frac{\gamma}{2}[d(x, T x)+d(y, T y)]
$$

Every Kannan contraction mapping on a complete metric space has a unique fixed point.

A self mapping $T$ on $(X, d)$ is called Boyd-Wong contraction if for all $x, y \in X$, we have

$$
d(T x, T y) \leq \phi(d(x, y)),
$$

where $\phi:[0, \infty) \rightarrow[0, \infty)$ is a continuous function satisfying $\phi(t)<t$ for every $t>0$.

Every Boyd-Wong contraction mapping on a complete metric space has a unique fixed point. 
Suppose that $x_{0}$ is an arbitrarily fixed element in $(X, d)$. Define $\|x\|=d\left(x, x_{0}\right)$. Let $\Lambda \geq 0, \lambda \geq 1$ and $\mu \in[0, \lambda]$ be fixed constants. Let $\psi:[0,1] \rightarrow[0, \infty)$ be an increasing function which is continuous at 0 and $\psi(0)=0$.

A self mapping $T$ on $(X, d)$ is called Pata contraction ([10]) if for all $x, y \in X$, we have

$$
d(T x, T y) \leq(1-\varepsilon) d(x, y)+\Lambda \varepsilon^{\lambda} \psi(\varepsilon)[1+\|x\|+\|y\|]^{\mu},
$$

for every $\varepsilon \in[0,1]$.

Every Pata contraction mapping on a complete metric space has a unique fixed point ([10]).

For comparison of Banach contraction principle and Boyd-Wong contraction theorem with Pata contraction theorem, we refer to ([10]).

A self mapping $T$ on $(X, d)$ is called Kanan Pata type contraction ([2]), if for all $x, y \in X$, we have

$$
\begin{aligned}
d(T x, T y) \leq & \frac{(1-\varepsilon)}{2}[d(x, T x)+d(y, T y)] \\
& +\Lambda \varepsilon^{\lambda} \Psi(\varepsilon)[1+\|x\|+\|y\|+\|T x\|+\|T y\|]^{\mu},
\end{aligned}
$$

for every $\varepsilon \in[0,1]$.

Every Kanan Pata type contraction mapping on a complete metric space has a unique fixed point ([2]).

It is known that Kanan Pata type contraction mapping theorem extends Kanan contraction theorem.

Balasubramanian ([1]) studied fixed point results of Chakraborty-Samanta contraction mapping (1.4) in the framework of complete cone metric space.

A self mapping $T$ on $(X, d)$ is called Chatterjea Pata type contraction ([8]), if for all $x, y \in X$, we have

$$
\begin{aligned}
& d(T x, T y) \leq \\
& \quad \frac{(1-\varepsilon)}{2}[d(x, T y)+d(y, T x)]+\Lambda \varepsilon^{\lambda} \psi(\varepsilon)[1+\|x\|+\|y\|+\|T x\|+\|T y\|]^{\mu},
\end{aligned}
$$

for every $\varepsilon \in[0,1]$.

Every Chatterjea Pata type contraction mapping on a complete metric space has a unique fixed point ([8]). For related results of Chatterjea Pata type contraction mapping and their comparison with comparable existing results in the literature, we refer to ([7], [8]).

Let $(A, B)$ be a pair of nonempty subsets of a metric space $(X, d)$. Define

$$
\begin{aligned}
& A_{0}=\{x \in A: d(x, y)=d(A, B) \text { for some } y \in B\}, \\
& B_{0}=\{y \in B: d(x, y)=d(A, B) \text { for some } x \in A\},
\end{aligned}
$$

where

$$
d(A, B)=\inf \{d(x, y): x \in A \text { and } y \in B\}
$$


If $A$ intersects with $B$, then $A \cap B$ is contained in both $A_{0}$ and $B_{0}$.

A set $B$ is said to be approximate compact with respect to $A$ if every sequence $\left\{y_{n}\right\}$ in $B$ satisfying $d\left(x, y_{n}\right) \longrightarrow d(x, B)$ has a convergent subsequence for some $x \in A$, where $d(x, A)=\inf _{a \in A} d(x, a)$.

It is evident that every set is approximate compact with respect to itself. Further, it can be seen that if $A$ is compact and $B$ is approximate compact with respect to $A$, then the sets $A_{0}$ and $B_{0}$ are nonempty.

The solution $x^{*}$ of the following optimization problem

$$
\inf _{x \in A} d(x, T x),
$$

is called approximate fixed point of $T$ or approximate solution of an operator equation $x=T x$.

Fan ([5]) studied the existence of approximate fixed points of a continuous mapping on a nonempty compact convex set $X$ in a Hausdorff locally convex topological vector space $E$.

An element $x^{*}$ in $A$ is called a best proximity point of $T$ if $d\left(x^{*}, T x^{*}\right)=d(A, B)$, where $A \cap B=\phi$. Note that $d(A, B)=0$, if $A \cap B \neq \phi$. Clearly, if $A=B$, then best proximity point of $T$ becomes a fixed point of $T$.

Let $T: A \rightarrow B$ and $g: A \longrightarrow A$. An element $x^{*}$ in $A$ is said to be optimal coincidence point of a pair $(g, T)$, if $d\left(g x^{*}, T x^{*}\right)=d(A, B)$ holds.

For best proximity point results of certain mappings, we refer to $([3,4,9,12])$ and reference mentioned therein.

From now onward we assume that $A, B$ are nonempty closed subsets of a complete metric space $(X, d)$.

Definition 1 ([13]). A mapping $f: X \rightarrow X$ is an expansive mapping if

$$
d(f x, f y) \geq q d(x, y),
$$

for all $q>1$ and $x, y \in X$.

Definition 2. Let $\alpha: X \times X \rightarrow[0, \infty)$. A self mapping $T$ on $X$ is said to be $\alpha$-admissible if

$$
\alpha(x, y) \geq 1 \text { implies that } \alpha(T x, T y) \geq 1 .
$$

Definition 3. Let $\alpha: X \times X \rightarrow[0, \infty)$. A sequence $\left\{x_{n}\right\}$ in $X$ is said to be $\alpha-$ regular if $\alpha\left(x_{n}, x_{n+1}\right) \geq 1$ for all $n \in \mathbb{N}$ and $\lim _{n \rightarrow \infty} x_{n}=x \in X$ imply that there exists a subsequence $\left\{x_{n_{k}}\right\}$ of $\left\{x_{n}\right\}$ such that $\alpha\left(x_{n_{k}}, x\right) \geq 1$ for all $k$.

Definition 4. Let $T: X \rightarrow X$ and $\alpha: X \times X \rightarrow[0, \infty)$. A mapping $T$ is said to be $\alpha$-proximal admissible if

$$
\left.\begin{array}{r}
\alpha(x, y) \geq 1 \\
d(u, T x)=d(A, B) \\
d(v, T y)=d(A, B)
\end{array}\right\} \operatorname{imply} \alpha(u, v) \geq 1,
$$


for all $x, y, u, v \in A$.

Definition 5. Let $\alpha: A \times A \rightarrow[0, \infty)$. A mapping $T: A \longrightarrow B$ is said to be a $\alpha-$ Pata-proximal admissible contraction of:

(a): type-I if for any $u, v, x$ and $y$ in $A$, there exist constants $\Lambda \geq 0, \lambda \geq 1$ and $\mu \in[0, \lambda]$ such that the following holds:

$$
\left.\begin{array}{r}
\alpha(x, y) \geq 1 \\
d(u, T x)=d(A, B) \\
d(v, T y)=d(A, B)
\end{array}\right\} \text { imply } \alpha(u, v) \geq 1
$$

and

$$
\alpha(x, y) d(u, v) \leq \frac{(1-\varepsilon)}{2} d(x, y)+\Lambda \varepsilon^{\lambda} \Psi(\varepsilon)[1+\|x\|+\|y\|+\|v\|]^{\mu},
$$

(b): type-II if for any $u, v, x$ and $y$ in $A$, there exist constants $\Lambda \geq 0, \lambda \geq 1$ and $\mu \in[0, \lambda]$ such that the following holds:

$$
\left.\begin{array}{r}
\alpha(x, y) \geq 1 \\
d(u, T x)=d(A, B) \\
d(v, T y)=d(A, B)
\end{array}\right\} \text { imply } \alpha(u, v) \geq 1
$$

and

$$
\alpha(x, y) d(T u, T v) \leq \frac{(1-\varepsilon)}{2} d(T x, T y)+\Lambda \varepsilon^{\lambda} \psi(\varepsilon)[1+\|T x\|+\|T y\|+\|T v\|]^{\mu} .
$$

for every $\varepsilon \in[0,1]$, where $\psi:[0,1] \rightarrow[0, \infty)$ is an increasing function which is continuous at 0 and $\psi(0)=0$.

Definition 6. Let $T: A \longrightarrow B, \alpha: A \times A \rightarrow[0, \infty)$ and $g: A \longrightarrow A$. A pair $(g, T)$ is said to be generalized $\alpha$-Pata-proximal contraction if for any $u, v, x$ and $y$ in $A$, there exist constants $\Lambda \geq 0, \lambda \geq 1$ and $\mu \in[0, \lambda]$ such that the following holds

$$
\left.\begin{array}{r}
\alpha(x, y) \geq 1 \\
d(g u, T x)=d(A, B) \\
d(g v, T y)=d(A, B)
\end{array}\right\} \text { imply } \alpha(g u, g v) \geq 1
$$

and

$$
\alpha(x, y) d(g u, g v) \leq \frac{(1-\varepsilon)}{2} d(x, y)+\Lambda \varepsilon^{\lambda} \psi(\varepsilon)[1+\|x\|+\|y\|+\|v\|]^{\mu}
$$

for every $\varepsilon \in[0,1]$, where $\psi:[0,1] \rightarrow[0, \infty)$ is an increasing function, which is continuous at 0 and $\psi(0)=0$.

Note that if $g=I_{A}$ (identity mapping on $A$ ), then generalized $\alpha$-Pata-proximal contraction becomes $\alpha$ - Pata-proximal admissible contraction of type-I.

From now and onward, we use the notation $\Delta$ for a set $\left\{\left(x_{n}, y_{n}\right) \in A_{0} \times A_{0}\right.$ : either $x_{n} \preceq y_{n}$ or $y_{n} \preceq x_{n}$, for all $\left.n \in \mathbb{N}\right\}$.

We start with the following result. 
Lemma 1. Suppose that $T$ is $\alpha$-proximal admissible and $T\left(A_{0}\right) \subseteq B_{0}$, where $A_{0}$ is a nonempty set in $A$. If there exists $x_{0}$ and $x_{1}$ in $A_{0}$ satisfying $d\left(x_{1}, T x_{0}\right)=d(A, B)$ and $\alpha\left(x_{0}, x_{1}\right) \geq 1$, then there exists a sequence $\left\{x_{n}\right\} \subset A_{0}$ such that

$$
d\left(x_{n+1}, T x_{n}\right)=d(A, B) \text { and } \alpha\left(x_{n}, x_{n+1}\right) \geq 1 \text { for all } n \in \mathbb{N} .
$$

Proof. Since $A_{0}$ is nonempty and $x_{0}, x_{1} \in A_{0}$ satisfying $d\left(x_{1}, T x_{0}\right)=d(A, B)$ and $\alpha\left(x_{0}, x_{1}\right) \geq 1$. As $T x_{1} \in T\left(A_{0}\right) \subseteq B_{0}$, there exists $x_{2} \in A_{0}$ such that $d\left(x_{2}, T x_{1}\right)=d(A, B)$. Thus

$$
\begin{aligned}
\alpha\left(x_{0}, x_{1}\right) & \geq 1 \\
d\left(x_{1}, T x_{0}\right) & =d(A, B) \text { and } \\
d\left(x_{2}, T x_{1}\right) & =d(A, B),
\end{aligned}
$$

implies that $\alpha\left(x_{1}, x_{2}\right) \geq 1$. Continuing this way, we obtain a sequence $\left\{x_{n}\right\} \subset A_{0}$ and it satisfies the condition (1.5).

Definition 7. A sequence $\left\{x_{n}\right\} \subset A_{0}$ satisfying the condition (1.5) is called proximal admissible Picard sequence provided that $\alpha\left(x_{n}, x_{1}\right) \geq 1$ and $\alpha\left(x_{n}, x_{0}\right) \geq 1$ for all $n$.

Definition 8. A set $A_{0}$ is called proximal $T$-orbitally complete if and only if every Cauchy proximal admissible Picard sequence in $A_{0}$ converges to an element in $A_{0}$.

We also need the following lemma in the sequel.

Lemma 2 ([6]). Let $A$ and $B$ be nonempty closed subsets of a metric space $(X, d)$. Suppose $A_{0}$ is a nonempty set and $B$ is approximately compact with respect to $A$. Then the set $A_{0}$ is closed.

Proposition 1. A self mapping $g: A \rightarrow A$ is said to satisfy $\alpha_{R}$-property if there exist a mapping $\alpha: A \times A \rightarrow[0, \infty)$ such that

$$
\alpha(g x, g y) \geq 1 \text { implies that } \alpha(x, y) \geq 1,
$$

for all $x, y \in A$.

\section{BEST PROXIMITY POINTS OF $\alpha$ - PATA-PROXIMAL ADMISSIBLE CONTRACTION MAPPINGS}

We start with the following lemma.

Lemma 3. Let $T: A \rightarrow B$ be a continuous and $\alpha$ - Pata-proximal admissible contraction of type-II mapping with $A_{0} \neq \varnothing$ and $T\left(A_{0}\right) \subseteq B_{0}$. Then $A_{0}$ is proximal $T$-orbitally complete. 
Proof. Let $x_{0} \in A_{0}$ and $\left\{x_{n}\right\}$ be a Cauchy proximal admissible Picard sequence. As $(X, d)$ is complete and $A$ is closed, there exist some $x^{*}$ in $A$ such that $\lim _{n \rightarrow \infty} d\left(x_{n}, x^{*}\right)=0$. By given assumption, we have

$$
\begin{aligned}
& d\left(x_{n}, T x_{n-1}\right)=d(A, B) \text { and } \\
& d\left(x_{n+1}, T x_{n}\right)=d(A, B) \text { with } \alpha\left(x_{n-1}, x_{n}\right) \geq 1,
\end{aligned}
$$

for all $n \in \mathbb{N}$. Since $T$ is a $\alpha$ - Pata-proximal admissible contraction of type-II, we have

$$
\begin{aligned}
d\left(T x_{n}, T x_{n+1}\right) \leq & \alpha\left(x_{n-1}, x_{n}\right) d\left(T x_{n}, T x_{n+1}\right) \\
\leq & \frac{(1-\varepsilon)}{2} d\left(T x_{n-1}, T x_{n}\right) \\
& +\Lambda \varepsilon^{\lambda} \Psi(\varepsilon)\left[1+\left\|T x_{n-1}\right\|+\left\|T x_{n}\right\|+\left\|T x_{n+1}\right\|\right]^{\mu} \\
< & d\left(T x_{n-1}, T x_{n}\right) \\
& +\Lambda \varepsilon^{\lambda} \Psi(\varepsilon)\left[1+\left\|T x_{n-1}\right\|+\left\|T x_{n}\right\|+\left\|T x_{n+1}\right\|\right]^{\mu} .
\end{aligned}
$$

On taking limit as $\varepsilon$ approaches to 0 , we have

$$
d\left(T x_{n}, T x_{n+1}\right) \leq d\left(T x_{n-1}, T x_{n}\right),
$$

which implies that $\left\{d\left(T x_{n-1}, T x_{n}\right)\right\}$ is a decreasing sequence and hence is convergent. Let $\lim _{n \rightarrow \infty} d\left(T x_{n+1}, T x_{n}\right)=r \geq 0$. We have to show that $r=0$. First we show that $\left\{d\left(T x_{n}, x_{0}\right)\right\}$ is bounded above by some constant $c$. Note that

$$
\begin{aligned}
d\left(T x_{n+1}, T x_{n}\right) & \leq d\left(T x_{n}, T x_{n-1}\right) \\
& \leq \cdots \\
& \leq d\left(T x_{1}, T x_{0}\right) \\
& \leq d\left(T x_{1}, x_{0}\right)+d\left(x_{0}, T x_{0}\right),
\end{aligned}
$$

for all $n=0,1,2, \ldots$. Let

$$
\left\|T x_{0}\right\|=c_{0} \text { and } c_{1}=\left\|T x_{1}\right\|
$$

for some $c_{0}, c_{1} \in \mathbb{R}^{+}$. Then

$$
\begin{aligned}
c_{n}=d\left(T x_{n}, x_{0}\right) \leq & d\left(T x_{n}, T x_{n+1}\right)+d\left(T x_{n+1}, T x_{1}\right)+d\left(T x_{1}, x_{0}\right) \\
\leq & \left(d\left(T x_{1}, x_{0}\right)+d\left(x_{0}, T x_{0}\right)\right)+d\left(T x_{n+1}, T x_{1}\right)+d\left(T x_{1}, x_{0}\right) \\
= & 2 d\left(T x_{1}, x_{0}\right)+d\left(x_{0}, T x_{0}\right)+d\left(T x_{n+1}, T x_{1}\right) \\
\leq & 2 d\left(T x_{1}, x_{0}\right)+d\left(x_{0}, T x_{0}\right)+\alpha\left(x_{n}, x_{0}\right) d\left(T x_{n+1}, T x_{1}\right) \\
\leq & 2 d\left(T x_{1}, x_{0}\right)+d\left(x_{0}, T x_{0}\right)+\frac{(1-\varepsilon)}{2} d\left(T x_{n}, T x_{0}\right) \\
& +\Lambda \varepsilon^{\lambda} \psi(\varepsilon)\left[1+\left\|T x_{0}\right\|+\left\|T x_{1}\right\|+\left\|T x_{n}\right\|\right]^{\mu}
\end{aligned}
$$




$$
\begin{aligned}
& \leq 2 c_{1}+c_{0}+\frac{(1-\varepsilon)}{2} c_{n}+\frac{(1-\varepsilon)}{2} c_{0}+\Lambda \varepsilon^{\lambda} \psi(\varepsilon)\left[1+c_{0}+c_{1}+c_{n}\right]^{\mu} \\
& \leq 2 c_{1}+2 c_{0}+c_{n}-\varepsilon c_{n}+\Lambda \varepsilon^{\lambda} \psi(\varepsilon) c_{n}^{\mu}\left[\frac{1+c_{0}+c_{1}}{c_{n}}+1\right]^{\mu}
\end{aligned}
$$

which further implies that

$$
\varepsilon c_{n} \leq 2 c_{1}+2 c_{0}+\Lambda \varepsilon^{\lambda} \psi(\varepsilon) c_{n}^{\lambda}\left[\frac{1+c_{0}+c_{1}}{c_{n}}+1\right]^{\mu},
$$

as $\mu \leq \lambda$. Suppose that $a_{n}=\Lambda\left[\frac{1+c_{0}+c_{1}}{c_{n}}+1\right]^{\mu}$ and $b=2\left(c_{1}+c_{0}\right)$. Then, the above inequality becomes

$$
\varepsilon c_{n} \leq \varepsilon^{\lambda} \psi(\varepsilon) c_{n}^{\lambda} a_{n}+b
$$

for some $a_{n}, b>0$. If there is a divergent subsequence $\left\{c_{n_{i}}\right\}$, correspondingly we have a subsequence $\left\{a_{n_{i}}\right\}$ such that $a_{n_{i}} \rightarrow \Lambda$. If we choose $\varepsilon_{n_{i}}=\frac{(1+b)}{c_{n_{i}}}$, then

$$
1 \leq \psi\left(\varepsilon_{i}\right)(1+b)^{\lambda} a_{n_{i}} \rightarrow 0,
$$

gives a contradiction. Now, we have to show that $\left\{T x_{n}\right\}$ is a Cauchy sequence. For this, we prove that

$$
d\left(T x_{n+m}, T x_{n}\right) \leq C \omega_{n}(\alpha),
$$

where $C=\sup _{n \in \mathbb{N}} \Lambda\left(1+3 c_{n}\right)^{\mu}<\infty$ and $\omega_{n}(\lambda)=\left(\frac{\lambda}{n}\right)^{\lambda} \sum_{k=1}^{n} \psi\left(\frac{\lambda}{k}\right)$.

Let $m$ be fixed, define

$$
p_{n}=n^{\lambda} d\left(T x_{n+m}, T x_{n}\right) \text {. }
$$

Note that

$$
\begin{aligned}
p_{n+1}= & (n+1)^{\lambda} d\left(T x_{n+m+1}, T x_{n+1}\right) \\
\leq & (n+1)^{\lambda} \alpha\left(x_{n+m}, x_{n}\right) d\left(T x_{n+m+1}, T x_{n+1}\right) \\
\leq & (n+1)^{\lambda} \frac{(1-\varepsilon)}{2} d\left(T x_{n+m}, T x_{n}\right) \\
& \left.+(n+1)^{\lambda} \Lambda \varepsilon^{\lambda} \psi(\varepsilon)\left[1+\left\|T x_{n+m}\right\|+\left\|T x_{n}\right\|+\left\|T x_{n+1}\right\|\right]^{\mu}\right] \\
\leq & (n+1)^{\lambda}(1-\varepsilon) d\left(T x_{n+m}, T x_{n}\right) \\
& \left.+(n+1)^{\lambda} \Lambda \varepsilon^{\lambda} \psi(\varepsilon)\left[1+\left\|T x_{n+m}\right\|+\left\|T x_{n}\right\|+\left\|T x_{n+1}\right\|\right]^{\mu}\right] .
\end{aligned}
$$

Choose

Then, we have

$$
\varepsilon=1-\left(\frac{n}{n+1}\right)^{\lambda}=\left[1-\left(1-\frac{1}{n+1}\right)^{\lambda}\right] \leq \frac{\lambda}{n+1} .
$$

$$
\begin{aligned}
p_{n+1} \leq & (n+1)^{\lambda}\left(\left(\frac{n}{n+1}\right)^{\lambda}\right) d\left(T x_{n+m}, T x_{n}\right) \\
& +(n+1)^{\lambda} \Lambda\left(\frac{\lambda}{n+1}\right)^{\lambda} \Psi\left(\frac{\lambda}{n+1}\right)\left[1+3 c_{n}\right]^{\mu}
\end{aligned}
$$




$$
\leq n^{\lambda} d\left(T x_{n+m}, T x_{n}\right)+\Lambda(\lambda)^{\lambda} \psi\left(\frac{\lambda}{n+1}\right)\left[1+3 c_{n}\right]^{\mu} .
$$

Now, we have

$$
\begin{aligned}
p_{n+1} \leq & p_{n}+C(\lambda)^{\lambda} \psi\left(\frac{\lambda}{n+1}\right) \\
\leq & p_{n-1}+C(\lambda)^{\lambda} \psi\left(\frac{\lambda}{n}\right)+C(\lambda)^{\lambda} \psi\left(\frac{\lambda}{n+1}\right) \\
& \ldots \\
\leq & p_{0}+C(\lambda)^{\lambda}\left[\psi\left(\frac{\lambda}{1}\right)+\psi\left(\frac{\lambda}{2}\right)+\cdots+\psi\left(\frac{\lambda}{n+1}\right)\right] .
\end{aligned}
$$

Since $p_{0}=0$, this gives

$$
p_{n} \leq C(\lambda)^{\lambda} \sum_{k=1}^{n} \psi\left(\frac{\lambda}{k}\right)
$$

After division by $n^{\lambda}$, we obtain that

$$
\frac{p_{n}}{n^{\lambda}}=d\left(T x_{n+m}, T x_{n}\right) \leq \frac{C(\lambda)^{\lambda}}{n^{\lambda}} \sum_{k=1}^{n} \psi\left(\frac{\lambda}{k}\right) .
$$

On taking as limit $n \rightarrow \infty,\left\{T x_{n}\right\}$ is a Cauchy sequence. For each $\varepsilon \in[0,1]$, we have

$$
\begin{aligned}
d\left(T x_{n}, T x_{n+1}\right) \leq & \alpha\left(x_{n-1}, x_{n}\right) d\left(T x_{n}, T x_{n+1}\right) \\
\leq & \frac{(1-\varepsilon)}{2} d\left(T x_{n-1}, T x_{n}\right) \\
& +\Lambda \varepsilon^{\lambda} \Psi(\varepsilon)\left[1+\left\|T x_{n-1}\right\|+\left\|T x_{n}\right\|+\left\|T x_{n+1}\right\|\right]^{\mu} \\
\leq & \frac{(1-\varepsilon)}{2} d\left(T x_{n-1}, T x_{n}\right)+\Lambda \varepsilon^{\lambda} \psi(\varepsilon)[1+3 c]^{\mu},
\end{aligned}
$$

for each $\varepsilon \in[0,1]$. Hence $\left\{T x_{n}\right\}$ is a Cauchy sequence in a complete metric space $(X, d)$ and $B$ is closed, there exists $y \in B$ such that

$$
\lim _{n \rightarrow \infty} d\left(T x_{n}, y\right)=0 \text {. }
$$

Since $\left\{x_{n}\right\}$ is Cauchy proximal admissible Picard sequence in $A_{0}$, and $x_{n} \rightarrow x^{*}$ in $A$ and mapping $T$ is continuous. Now, from the above inequality, we have

$$
d(A, B)=\lim _{n \rightarrow \infty} d\left(x_{n+1}, T x_{n}\right)=d\left(x^{*}, y\right),
$$

which implies that $x^{*} \in A_{0}$.

Theorem 1. Let $T: A \rightarrow B$ be a continuous $\alpha-$ proximal admissible and $\alpha-$ Pata-proximal admissible contraction of type-II, with $A_{0} \neq \phi$ and $T\left(A_{0}\right) \subseteq B_{0}$. If $B$ is approximately compact with respect to $A$, then $T$ has a unique best proximity point $x^{*}$ in $A_{0}$. 
Proof. Let $x_{0}$ be a given point in $A_{0}$. From Lemma (1), sequence $\left\{x_{n}\right\}$ is Cauchy proximal admissible Picard sequence in $A_{0}$. We can obtain a sequence $\left\{x_{n}\right\}$ in $A_{0}$ such that it satisfies

$$
d\left(x_{n}, T x_{n-1}\right)=d(A, B), d\left(x_{n+1}, T x_{n}\right)=d(A, B) \text { and } \alpha\left(x_{n-1}, x_{n}\right) \geq 1
$$

for all $n \in \mathbb{N}$. Since $T$ is $\alpha$-proximal admissible and $\alpha$ - Pata-proximal admissible contraction of type-II. Following arguments similar to those in the proof of Lemma (3), we obtain that $\left\{T x_{n}\right\} \in B_{0}$ is a Cauchy proximal admissible Picard sequence. As $B$ is a closed subset of a complete metric space, there exists some $y \in B$ such that $\lim _{n \rightarrow \infty} d\left(T x_{n}, y\right)=0$. Since $A_{0}$ is proximal $T$-orbitally complete, there exists some $x^{*}$ in $A_{0}$ such that $x_{n} \rightarrow x^{*}$ in $A_{0}$ which implies that $T x^{*}=y=\lim _{n \rightarrow \infty} T x_{n}$ and hence

$$
d\left(x^{*}, T x^{*}\right)=d(A, B),
$$

implies that $x^{*}$ is the Pata-type best proximity point of the mapping $T$.

To show the uniqueness of best proximity point: Assume on contrary that there exists another point $y^{*} \neq x^{*}$ of $T$ in $A_{0}$ such that

$$
d\left(y^{*}, T y^{*}\right)=d(A, B), d\left(x^{*}, T x^{*}\right)=d(A, B) \text { and } \alpha\left(x^{*}, y^{*}\right) \geq 1 \text { where } x^{*}, y^{*} \in A_{0} .
$$

Since $T$ is $\alpha$ - Pata-proximal admissible contraction of type-II, so

$$
\begin{aligned}
\alpha\left(x^{*}, y^{*}\right) d\left(T x^{*}, T y^{*}\right) \leq & \frac{(1-\varepsilon)}{2} d\left(T x^{*}, T y^{*}\right) \\
& +\Lambda \varepsilon^{\lambda} \psi(\varepsilon)\left[1+\left\|T x^{*}\right\|+\left\|T y^{*}\right\|+\left\|T y^{*}\right\|\right]^{\mu} \\
\leq & \frac{(1-\varepsilon)}{2} d\left(T x^{*}, T y^{*}\right)+\Lambda \varepsilon^{\lambda} \Psi(\varepsilon)[1+3 c]^{\mu} \\
< & d\left(T x^{*}, T y^{*}\right)+\Lambda \varepsilon^{\lambda} \psi(\varepsilon)[1+3 c]^{\mu} .
\end{aligned}
$$

On taking limit as $\varepsilon \rightarrow 0^{+}$, we have

$$
\alpha\left(x^{*}, y^{*}\right) d\left(T x^{*}, T y^{*}\right)<d\left(T x^{*}, T y^{*}\right),
$$

a contradiction. Hence Pata-type best proximity point of the mapping $T$ is unique.

Example 1. Let $X=\mathbb{R}$. We know that $X$ is a complete metric space with respect to usual metric $d: X \times X \rightarrow[0, \infty)$ defined by $d(x, y)=|x-y|$. Let $A=\left\{0, \frac{2}{6}, \frac{4}{6}, \frac{6}{6}\right\}$ and $B=\left\{\frac{1}{6}, \frac{3}{6}, \frac{5}{6}, \frac{7}{6}, \frac{9}{6}, \frac{11}{6}, \frac{13}{6}\right\}$ be two closed subsets of $X$. Note that $B$ is compact and so $B$ is approximately compact with respect to $A$. Note that

$$
d(A, B)=\frac{1}{6}, A_{0}=A \text { and } B_{0}=\left\{\frac{1}{6}, \frac{3}{6}, \frac{5}{6}, \frac{7}{6}\right\} .
$$


Define a mapping $T: A \rightarrow B$ as:

$$
T x=\left\{\begin{array}{l}
2 x+\frac{1}{6} \text { when } x \in\left\{0, \frac{2}{6}\right\}, \\
2 x-\frac{1}{6} \text { when } x=\frac{4}{6}, \\
\frac{3}{6} \quad \text { when } x=\frac{6}{6} .
\end{array} .\right.
$$

Obviously, $T\left(A_{0}\right) \subseteq B_{0}$. Further, suppose that $\alpha(x, y)=e^{|x-y|}$ for all $x, y \in X$. Also, $\psi(t)=t e^{t}$ for all $t \geq 0$. If $\lambda=2$ then $\mu \in[0,2]$, take $\mu=\lambda$. For simplicity, take $\varepsilon=\frac{1}{10}$. If, we take $u=\frac{2}{6}, v=\frac{6}{6}, x=0$ and $y=\frac{2}{6}$ in $A$, satisfies the following:

$$
d(u, T x)=d(A, B), \text { and } d(v, T y)=d(A, B),
$$

Now, It is straightforward to check that mapping $T$ satisfy the $\alpha$ - Pata-proximal admissible contraction of type-II for $50.79922618 \leq \Lambda$. Assume, $\Lambda=51$. Moreover $x=0$ is the only Pata type best proximity point of mapping $T$.

\section{OPTIMAL COINCIDENCE POINT OF GENERALIZED $\alpha$ - PATA-PROXIMAL CONTRACTION MAPPINGS}

Theorem 2. Let $g: A \rightarrow A$ be an expansive mapping satisfying $\alpha_{R}$-property and $T: A \rightarrow B$ be an $\alpha$-proximal admissible with $A_{0} \neq \phi, T\left(A_{0}\right) \subseteq B_{0}$ and $A_{0} \subseteq g\left(A_{0}\right)$ for any $t>0$. If $B$ is approximately compact with respect to $A$ and the pair $(g, T)$ is generalized $\alpha$-Pata-proximal contraction. If there exists $x_{0}, x_{1} \in A_{0}$ satisfying $d\left(x_{1}, T x_{0}\right)=d(A, B)$ and $\alpha\left(x_{0}, x_{1}\right) \geq 1$. Then the pair $(g, T)$ has a unique optimal coincidence point $x^{*}$ in $A_{0}$.

Proof. Let $x_{0}$ be a given point in $A_{0}$. As $T\left(A_{0}\right) \subseteq B_{0}$ and $A_{0} \subseteq g\left(A_{0}\right)$, we can choose an element $x_{1} \in A_{0}$ such that $d\left(g x_{1}, T x_{0}\right)=d(A, B)$ and $\alpha\left(x_{0}, x_{1}\right) \geq 1$, where $x_{0}, x_{1} \in A_{0}$. Also, $T x_{1} \in T\left(A_{0}\right) \subseteq B_{0}$, and $A_{0} \subseteq g\left(A_{0}\right)$, it follows that there exists an element $x_{2} \in A_{0}$ such that $d\left(g x_{2}, T x_{1}\right)=d(A, B)$, since $T$ is $\alpha$-proximal admissible mapping then $\alpha\left(g x_{1}, g x_{2}\right) \geq 1$ further $g$ is satisfying $\alpha_{R}$-property, which implies that $\alpha\left(x_{1}, x_{2}\right) \geq 1$, where $g x_{1}, g x_{2} \in A_{0}$. Continuing this way, we can obtain a sequence $\left\{g x_{n}\right\}$ in $A_{0}$ satisfying:

$$
d\left(g x_{n}, T x_{n-1}\right)=d(A, B) \text { and } d\left(g x_{n+1}, T x_{n}\right)=d(A, B),
$$

where $\alpha\left(x_{n-1}, x_{n}\right) \geq 1$. Note that

$$
\begin{aligned}
\alpha\left(x_{n-1}, x_{n}\right) d\left(g x_{n}, g x_{n+1}\right) \leq & \frac{(1-\varepsilon)}{2} d\left(x_{n-1}, x_{n}\right) \\
& +\Lambda \varepsilon^{\lambda} \psi(\varepsilon)\left[1+\left\|x_{n-1}\right\|+\left\|x_{n}\right\|+\left\|x_{n+1}\right\|\right]^{\mu} .
\end{aligned}
$$

Suppose that $x_{n} \neq x_{n+1}$ for all $n=0,1,2, \ldots$ For each $n \in \mathbb{N}$ and $\varepsilon \in[0,1]$,

$$
\begin{aligned}
d\left(x_{n}, x_{n+1}\right) & \leq \alpha\left(x_{n-1}, x_{n}\right) d\left(g x_{n}, g x_{n+1}\right) \\
& \leq \frac{(1-\varepsilon)}{2} d\left(x_{n-1}, x_{n}\right)
\end{aligned}
$$




$$
+\Lambda \varepsilon^{\lambda} \psi(\varepsilon)\left[1+\left\|x_{n-1}\right\|+\left\|x_{n}\right\|+\left\|x_{n+1}\right\|\right]^{\mu} .
$$

Thus, for each $n \in \mathbb{N}$, letting $\varepsilon \rightarrow 0^{+}$on both sides of the inequality (3.3), gives that

$$
\begin{aligned}
d\left(x_{n+1}, x_{n}\right) & \leq d\left(g x_{n+1}, g x_{n}\right) \\
& \leq \alpha\left(x_{n-1}, x_{n}\right) d\left(g x_{n+1}, g x_{n}\right) \leq \frac{d\left(x_{n}, x_{n-1}\right)}{2}<d\left(x_{n}, x_{n-1}\right) .
\end{aligned}
$$

Thus $\left\{x_{n}\right\}$ is decreasing sequence. Continuing this way, we have

$$
d\left(x_{n+1}, x_{n}\right) \leq d\left(x_{n}, x_{n-1}\right) \leq \cdots \leq d\left(x_{1}, x_{0}\right),
$$

for all $n=0,1,2, \ldots$.

Now, we have to show that the sequence $d\left(g x_{n}, x_{0}\right)$ is bounded above by $\frac{c}{2}=$ $d\left(x_{0}, g x_{1}\right)$. This is the case when $n=1$. Assume that $d\left(g x_{n-1}, x_{0}\right) \leq \frac{c}{2}$. We show that $d\left(g x_{n}, x_{0}\right) \leq c$. Note that

$$
\begin{aligned}
d\left(g x_{n}, x_{0}\right) & \leq d\left(g x_{n}, g x_{n-1}\right)+d\left(g x_{n-1}, x_{0}\right) \\
& \leq d\left(x_{n-1}, x_{n-2}\right)+d\left(g x_{n-1}, x_{0}\right) \\
& \leq \cdots \\
& \leq d\left(x_{1}, x_{0}\right)+\frac{c}{2} \\
& \leq \frac{c}{2}+\frac{c}{2}=c .
\end{aligned}
$$

Thus, for all $n \in \mathbb{N}$,

$$
d\left(g x_{n}, x_{0}\right) \leq c .
$$

Let

$$
\left\|g x_{0}\right\|=c_{0} \text { and } c_{1}=\left\|g x_{1}\right\|
$$

for some $c_{0}, c_{1} \in \mathbb{R}^{+}$. Then

$$
\begin{aligned}
c_{n}=d\left(g x_{n}, x_{0}\right) \leq & d\left(g x_{n}, g x_{n+1}\right)+d\left(g x_{n+1}, g x_{1}\right)+d\left(g x_{1}, x_{0}\right) \\
\leq & d\left(x_{n-1}, x_{n}\right)+d\left(g x_{n+1}, g x_{1}\right)+d\left(g x_{1}, x_{0}\right) \\
& \cdots \\
\leq & d\left(x_{0}, x_{1}\right)+d\left(g x_{n+1}, g x_{1}\right)+d\left(g x_{1}, x_{0}\right) \\
\leq & d\left(x_{0}, x_{1}\right)+d\left(g x_{1}, x_{0}\right)+d\left(g x_{n+1}, g x_{1}\right) \\
\leq & d\left(x_{0}, x_{1}\right)+d\left(g x_{1}, x_{0}\right)+\alpha\left(x_{n}, x_{0}\right) d\left(g x_{n+1}, g x_{1}\right) \\
\leq & d\left(x_{0}, x_{1}\right)+d\left(g x_{1}, x_{0}\right)+\frac{(1-\varepsilon)}{2} d\left(x_{n}, x_{0}\right) \\
& +\Lambda \varepsilon^{\lambda} \Psi(\varepsilon)\left[1+\left\|x_{0}\right\|+\left\|x_{1}\right\|+\left\|x_{n}\right\|\right]^{\mu} \\
\leq & \frac{c_{1}}{2}+c_{0}+\frac{(1-\varepsilon)}{2} c_{n}+\Lambda \varepsilon^{\lambda} \psi(\varepsilon)\left[1+c_{0}+c_{1}+c_{n}\right]^{\mu} \\
\leq & \frac{c_{1}}{2}+c_{0}+(1-\varepsilon) c_{n}+\Lambda \varepsilon^{\lambda} \psi(\varepsilon)\left[1+c_{0}+c_{1}+c_{n}\right]^{\mu}
\end{aligned}
$$




$$
\leq \frac{c_{1}}{2}+c_{0}+c_{n}-\varepsilon c_{n}+\Lambda \varepsilon^{\lambda} \psi(\varepsilon) c_{n}^{\mu}\left[\frac{1+c_{0}+c_{1}}{c_{n}}+1\right]^{\mu},
$$

which further implies that

$$
\varepsilon c_{n} \leq \frac{c_{1}}{2}+c_{0}+\Lambda \varepsilon^{\lambda} \psi(\varepsilon) c_{n}^{\lambda}\left[\frac{1+c_{0}+c_{1}}{c_{n}}+1\right]^{\mu},
$$

as $\mu \leq \lambda$. Suppose that $a_{n}=\Lambda\left[\frac{1+c_{0}+c_{1}}{c_{n}}+1\right]^{\mu}$ and $b=\frac{c_{1}}{2}+c_{0}$. Then, the above inequality becomes

$$
\varepsilon c_{n} \leq \varepsilon^{\lambda} \psi(\varepsilon) c_{n}^{\lambda} a_{n}+b
$$

for some $a_{n}, b>0$. If there is a divergent subsequence $\left\{c_{n_{i}}\right\}$, correspondingly, we have a subsequence $\left\{a_{n_{i}}\right\}$ such that $a_{n_{i}} \rightarrow \Lambda$. If we choose $\varepsilon_{n_{i}}=\frac{(1+b)}{c_{n_{i}}}$, then

$$
1 \leq \psi\left(\varepsilon_{i}\right)(1+b)^{\lambda} a_{n_{i}} \rightarrow 0,
$$

gives a contradiction.

Now, we have to show that $\left\{g x_{n}\right\}$ is Cauchy sequence. For this, we prove that

$$
d\left(g x_{n+m}, g x_{n}\right) \leq C \omega_{n}(\alpha),
$$

where $C=\sup _{n \in \mathbb{N}} \Lambda\left(1+3 c_{n}\right)^{\mu}<\infty$ and $\omega_{n}(\lambda)=\left(\frac{\lambda}{n}\right)^{\lambda} \sum_{k=1}^{n} \psi\left(\frac{\lambda}{k}\right)$.

Let $m$ be fixed, define

Note that

$$
p_{n}=n^{\lambda} d\left(g x_{n+m}, g x_{n}\right)
$$

$$
\begin{aligned}
p_{n+1}= & (n+1)^{\lambda} d\left(g x_{n+m+1}, g x_{n+1}\right) \\
\leq & (n+1)^{\lambda} \alpha\left(x_{n+m}, x_{n}\right) d\left(g x_{n+m+1}, g x_{n+1}\right) \\
\leq & (n+1)^{\lambda}\left[\frac{(1-\varepsilon)}{2} d\left(x_{n+m}, x_{n}\right)+\Lambda \varepsilon^{\lambda} \Psi(\varepsilon)\left[1+\left\|x_{n+m}\right\|+\left\|x_{n}\right\|+\left\|x_{n+1}\right\|\right]^{\mu}\right] \\
\leq & (n+1)^{\lambda}(1-\varepsilon) d\left(x_{n+m}, x_{n}\right) \\
& \left.+(n+1)^{\lambda} \Lambda \varepsilon^{\lambda} \Psi(\varepsilon)\left[1+\left\|x_{n+m}\right\|+\left\|x_{n}\right\|+\left\|x_{n+1}\right\|\right]^{\mu}\right] .
\end{aligned}
$$

Choose

Then, we have

$$
\varepsilon=1-\left(\frac{n}{n+1}\right)^{\lambda}=\left[1-\left(1-\frac{1}{n+1}\right)^{\lambda}\right] \leq \frac{\lambda}{n+1} .
$$

$$
\begin{aligned}
p_{n+1} & \leq(n+1)^{\lambda}\left(\left(\frac{n}{n+1}\right)^{\lambda}\right) d\left(x_{n+m}, x_{n}\right)+(n+1)^{\lambda} \Lambda\left(\frac{\lambda}{n+1}\right)^{\lambda} \Psi\left(\frac{\lambda}{n+1}\right)\left[1+3 c_{n}\right]^{\mu} \\
& \leq n^{\lambda} d\left(x_{n+m}, x_{n}\right)+\Lambda(\lambda)^{\lambda} \Psi\left(\frac{\lambda}{n+1}\right)\left[1+3 c_{n}\right]^{\mu} \\
& \leq n^{\lambda} d\left(g x_{n+m}, g x_{n}\right)+\Lambda(\lambda)^{\lambda} \Psi\left(\frac{\lambda}{n+1}\right)\left[1+3 c_{n}\right]^{\mu} .
\end{aligned}
$$


Now, we have

$$
\begin{aligned}
p_{n+1} \leq & p_{n}+C(\lambda)^{\lambda} \Psi\left(\frac{\lambda}{n+1}\right) \\
\leq & p_{n-1}+C(\lambda)^{\lambda} \Psi\left(\frac{\lambda}{n}\right)+C(\lambda)^{\lambda} \Psi\left(\frac{\lambda}{n+1}\right) \\
& \ldots \\
\leq & p_{0}+C(\lambda)^{\lambda}\left[\psi\left(\frac{\lambda}{1}\right)+\psi\left(\frac{\lambda}{2}\right)+\cdots+\psi\left(\frac{\lambda}{n+1}\right)\right] .
\end{aligned}
$$

Now, $p_{0}=0$ gives that

$$
p_{n} \leq C(\lambda)^{\lambda} \sum_{k=1}^{n+1} \psi\left(\frac{\lambda}{k}\right)
$$

After division by $n^{\lambda}$, we will obtain

$$
\frac{p_{n}}{n^{\lambda}}=d\left(g x_{n+m}, g x_{n}\right) \leq \frac{C(\lambda)^{\lambda}}{n^{\lambda}} \sum_{k=1}^{n} \psi\left(\frac{\lambda}{k}\right) .
$$

On taking limit as $n \rightarrow \infty$, we have $\left\{g x_{n}\right\}$, a Cauchy sequence. For each $\varepsilon \in[0,1]$, we have

$$
\begin{aligned}
d\left(g x_{n}, g x_{n+1}\right) & \leq \alpha\left(x_{n-1}, x_{n}\right) d\left(g x_{n}, g x_{n+1}\right) \\
& \leq \frac{(1-\varepsilon)}{2} d\left(x_{n-1}, x_{n}\right)+\Lambda \varepsilon^{\lambda} \Psi(\varepsilon)\left[1+\left\|x_{n-1}\right\|+\left\|x_{n}\right\|+\left\|x_{n+1}\right\|\right]^{\mu}, \\
& \leq \frac{(1-\varepsilon)}{2} d\left(x_{n-1}, x_{n}\right)+\Lambda \varepsilon^{\lambda} \Psi(\varepsilon)[1+3 c]^{\mu},
\end{aligned}
$$

for each $\varepsilon \in[0,1]$. Hence $\left\{g x_{n}\right\}$ is a Cauchy sequence in a complete metric space $(X, d)$. Since $A_{0}$ is closed (Lemma 2), there exists an element $x^{*}$ in $A_{0}$ such that $\lim _{n \rightarrow \infty} d\left(g x_{n}, g x^{*}\right)=0$. Now

$$
\begin{aligned}
d\left(g x^{*}, B\right) & \leq d\left(g x^{*}, T x_{n}\right) \\
& \leq d\left(g x^{*}, g x_{n+1}\right)+d\left(g x_{n+1}, T x_{n}\right) \\
& =d\left(g x^{*}, g x_{n+1}\right)+d(A, B) \\
& \leq d\left(g x^{*}, g x_{n+1}\right)+d\left(g x^{*}, B\right),
\end{aligned}
$$

gives that

$$
d\left(g x^{*}, B\right) \leq d\left(g x^{*}, T x_{n}\right) \leq d\left(g x^{*}, g x_{n+1}\right)+d\left(g x^{*}, B\right) .
$$

Note that $\left\{g x_{n}\right\}$ converges to $g x^{*}$ and $d\left(g x^{*}, T x_{n}\right) \rightarrow d\left(g x^{*}, B\right)$. As $\left\{T x_{n}\right\} \subseteq B$ and $B$ is approximately compact with respect to $A,\left\{T x_{n}\right\}$ has a subsequence, which converges to some $y$ in $B$ hence $d\left(g x^{*}, y\right)=d(A, B)$, that is, $g x^{*} \in A_{0}$. Since $A_{0} \subseteq g\left(A_{0}\right)$, there exist some $u \in A_{0}$ such that

$$
d\left(g u, T x^{*}\right)=d(A, B)=d\left(g x_{n+1}, T x_{n}\right),
$$


where $x^{*}, x_{n} \in A_{0}, \alpha\left(x^{*}, u\right) \geq 1$ for all $n \in \mathbb{N}$. Since $g$ is expansive mapping, we have to show that $u=x^{*}$. If not, then

$$
\begin{aligned}
d\left(u, x_{n+1}\right) & \leq \alpha\left(x^{*}, x_{n}\right) d\left(g u, g x_{n+1}\right) \\
& \leq \frac{(1-\varepsilon)}{2} d\left(x^{*}, x_{n}\right)+\Lambda \varepsilon^{\lambda} \psi(\varepsilon)\left[1+\left\|x^{*}\right\|+\left\|x_{n}\right\|+\left\|x_{n+1}\right\|\right]^{\mu},
\end{aligned}
$$

on taking limit as $n \rightarrow \infty$, and $\varepsilon \rightarrow 0^{+}$gives

$$
d\left(u, x^{*}\right) \leq \alpha\left(x^{*}, x^{*}\right) d\left(g u, g x^{*}\right) \leq 0
$$

a contradiction. Hence $d\left(g x^{*}, T x^{*}\right)=d\left(g u, T x^{*}\right)=d(A, B)$, that is, $x^{*}$ is the optimal coincidence point of the pair $(g, T)$.

To prove uniqueness: Suppose that $y^{*}$ be another point in $A_{0}$ such that $d\left(g y^{*}, T y^{*}\right)$ $=d(A, B)$, such that $\alpha\left(x^{*}, y^{*}\right) \geq 1$. Note that

$$
\begin{aligned}
d\left(g x^{*}, g y^{*}\right) & \leq \alpha\left(x^{*}, y^{*}\right) d\left(g x^{*}, g y^{*}\right) \\
& \leq \frac{(1-\varepsilon)}{2} d\left(x^{*}, y^{*}\right)+\Lambda \varepsilon^{\lambda} \psi(\varepsilon)\left[1+\left\|x^{*}\right\|+\left\|x^{*}\right\|+\left\|x^{*}\right\|\right]^{\mu},
\end{aligned}
$$

and hence

$$
d\left(x^{*}, y^{*}\right) \leq d\left(g x^{*}, g y^{*}\right) \leq \frac{(1-\varepsilon)}{2} d\left(x^{*}, y^{*}\right)+\Lambda \varepsilon^{\lambda} \psi(\varepsilon)\left[1+\left\|x^{*}\right\|+\left\|x^{*}\right\|+\left\|x^{*}\right\|\right]^{\mu} .
$$

Apply $\varepsilon \rightarrow 0^{+}$, then the above inequality gives a contradiction. The result follows.

Example 2. Let $X=\mathbb{R}^{2}$. We know that $X$ is a complete metric space with respect to usual metric $d: X \times X \rightarrow[0, \infty)$ defined by $d(x, y)=|x-y|$. Let $A=\{(x, 2) \mid x \in \mathbb{R}$ \} and $B=\{(x, 0) \mid x \in \mathbb{R}$ and $x \geq-3\}$ be two closed subsets of $X$. Note that $B$ is compact and so $B$ is approximately compact with respect to $A$. Note that $d(A, B)=$ $2, A_{0}=\{(x, 2) \mid x \in \mathbb{R}$ and $x \geq-3\} \subset A$ and $B_{0}=\{(x, 0) \mid x \in \mathbb{R}$ and $x \geq-3\} \subset B$. Define a mapping $T: A \rightarrow B$ as:

$$
T(x, 2)=(2 x+3,0) \text { and } g(x, 2)=(3 x, 2) .
$$

Obviously, $T\left(A_{0}\right) \subseteq B_{0}$. Further, suppose that $\alpha(x, y)=e^{|x-y|}$ and $\psi(t)=t e^{t}$ for all $x, y \in X$ and $t \geq 0$. If, we choose $\lambda=1$ then $\mu \in[0,1]$ take $\mu=\lambda$. For simplicity, take $x_{0}=(0,2)$ and $\varepsilon=\frac{1}{10}$. If $u=\left(u_{1}, 2\right), v=\left(v_{1}, 2\right), x=\left(x_{1}, 2\right)$ and $y=\left(y_{1}, 2\right) \in A$ satisfies:

$$
d(g u, T x)=d(A, B) \text { and } d(g v, T y)=d(A, B) .
$$

Which implies that

$$
u_{1}=\frac{2}{3} x_{1}+1 \text { and } v_{1}=\frac{2}{3} y_{1}+1 .
$$

Now, it is straightforward to check that the pair $(g, T)$ satisfies the generalized $\alpha-$ Pata-proximal admissible contraction for $\frac{140.2498021\left|x_{1}-y_{1}\right|}{\left|1+x_{1}+y_{1}+v_{1}\right|} \leq \Lambda$, where $\frac{\left|x_{1}-y_{1}\right|}{\left|1+x_{1}+y_{1}+v_{1}\right|} \leq 1$ for all $x_{1}, y_{1}, v_{1} \in A_{0}$ and $x_{1} \neq y_{1}$, hence $140.2498021 \leq \Lambda$. Assume, 
$\Lambda=141$. Moreover $x=(-3,2)$ is the only Pata-type coincidence best proximity point of pair $(g, T)$.

Corollary 1. If $T: A \rightarrow B$ is $\alpha$-Pata-proximal contraction of type-I, $\alpha$-proximal admissible with $A_{0} \neq \phi$ and $T\left(A_{0}\right) \subseteq B_{0}$ for any $t>0$. Then $T$ has a unique best proximity point $x^{*}$ in $A_{0}$ provided that $A_{0}$ is proximal $T$-orbitally complete.

Proof. Take $g x=I_{A}$ in the proof of Theorem (2).

Example 3. Let $X=\mathbb{R}^{2}$. We know $X$ is a complete metric space with respect to usual metric $d: X \times X \rightarrow[0, \infty)$ defined as $d(x, y)=|x-y|$. Let $A=\{(x, 1) \mid x \in \mathbb{R}$ and $x \geq-1\}$ and $B=\{(x, 0) \mid x \in \mathbb{R}$ and $x \geq-3\}$ be two closed subsets of $X$. Note that, $B$ is compact and so $B$ is approximately compact with respect to $A$. Note that

$$
d(A, B)=1, A_{0}=A \text { and } B_{0}=\{(x, 0) \mid x \in \mathbb{R} \text { and } x \geq-1\} \subset B .
$$

Define a mapping $T: A \rightarrow B$ as:

$$
T(x, 1)=(2 x+1,0) .
$$

Obviously, $T\left(A_{0}\right) \subseteq B_{0}$. Further, suppose that $\alpha(x, y)=e^{|x-y|}$ for all $x, y \in X$ and $\psi(t)=t e^{t}$ for all $t \geq 0$. If, we choose $\lambda=1$ then $\mu \in[0,1]$ take $\mu=\lambda$. For simplicity, take $x_{0}=(0,1)$ and $\varepsilon=\frac{1}{10}$. If $u=\left(u_{1}, 1\right), v=\left(v_{1}, 1\right), x=\left(x_{1}, 1\right)$ and $y=\left(y_{1}, 1\right) \in A$ satisfies the following

$$
d(u, T x)=d(A, B) \text { and } d(v, T y)=d(A, B),
$$

if

$$
u_{1}=2 x_{1}+1 \text { and } v_{1}=2 y_{1}+1 .
$$

Now, It is straight forward to check that $T$ is $\alpha$ - Pata-proximal admissible contraction of type-I for $\frac{140.2498021\left|x_{1}-y_{1}\right|}{\left|1+x_{1}+y_{1}+v_{1}\right|} \leq \Lambda$, where $\frac{\left|x_{1}-y_{1}\right|}{\left|1+x_{1}+y_{1}+v_{1}\right|} \leq 1$ for all $x_{1}, y_{1}, v_{1} \in A_{0}$ and $x_{1} \neq y_{1}$, hence $140.2498021 \leq \Lambda$. Assume that $\Lambda=141$ and after simple calculation one can find that $x=(-1,1)$ is the only Pata type best proximity point of mapping $T$.

\section{APPROXIMATION RESUlTS IN ORDERED STRUCTURES}

In this section, we prove results in ordered metric spaces.

From now onwards, a 3 - tuple $(X, d, \preceq)$ is called a partially ordered metric space if $(X, \preceq)$ is a partially ordered set and $(X, d)$ is a metric space. Unless otherwise stated, it is assumed that $A, B$ are nonempty closed subsets of partially ordered metric space $(X, d, \preceq)$.

Definition 9 ([11]). A mapping $T: A \longrightarrow B$ is called (a) nondecreasing or order preserving if for any $x, y$ in $A$ with $x \preceq y$, we have $T x \preceq T y$ (b) nonincreasing or ordered reversing if for any $x, y$ in $A$ with $x \preceq y$, we have $T x \succeq T y$ (c) monotone if it is order preserving or order reversing. 
Definition 10 ([11]). A mapping $T: A \longrightarrow B$ is said to be a ordered contraction if for any $x, y \in A$ with $x \preceq y$, we have $d(T x, T y) \leq d(x, y)$.

Definition 11 ([11]). A mapping $T: A \longrightarrow B$ is said to be a proximal order preserving if for any $u, v, x$ and $y$ in $A$, the following implication holds:

$$
\left.\begin{array}{r}
x \preceq y \\
d(u, T x)=d(A, B) \\
d(v, T y)=d(A, B)
\end{array}\right\} \text { imply } u \preceq v .
$$

If $A=B$, then proximal order preserving mapping will become order preserving.

Definition 12 ([11]). A mapping $T: A \longrightarrow B$ is said to be a proximal order reversing if for any $u, v, x$ and $y$ in $A$, the following implication holds:

$$
\left.\begin{array}{r}
x \preceq y \\
d(u, T x)=d(A, B) \\
d(v, T y)=d(A, B)
\end{array}\right\} \text { imply } u \succeq v .
$$

If $A=B$, then proximal order reversing mapping will become order reversing. We start with the following result.

Lemma 4 ([11]). If $A_{0} \neq \phi$ and $T\left(A_{0}\right) \subseteq B_{0}$. Then, for $a \in A_{0}$, there exists $a$ sequence $\left\{x_{n}\right\} \subset A_{0}$ such that

$$
\left.\begin{array}{l}
x_{0}=a, \\
d\left(x_{n+1}, T x_{n}\right)=d(A, B), \text { for all } n \in \mathbb{N} \text { with }\left(x_{n}, x_{n+1}\right) \in \Delta .
\end{array}\right\}
$$

Proof. As $x_{0}=a \in A_{0}$ and $T\left(A_{0}\right) \subseteq B_{0}$, there exist $x_{1} \in A_{0}$ such that $d\left(x_{1}, T x_{0}\right)=d(A, B)$ where $\left(x_{0}, x_{1}\right) \in \Delta$. Also $T x_{0} \in T\left(A_{0}\right) \subseteq B_{0}$, there exist $x_{2} \in A_{0}$, such that $d\left(x_{2}, T x_{1}\right)=d(A, B)$ where $\left(x_{1}, x_{2}\right) \in \Delta$. Continuing this way, we can obtain a sequence $\left\{x_{n}\right\} \subset A_{0}$ that satisfies the condition (4.1).

Definition 13. A sequence $\left\{x_{n}\right\} \subset A_{0}$ satisfying the condition (4.1) is called ordered proximal Picard sequence starting with $a \in A_{0}$.

Definition 14. A set $A_{0}$ is ordered proximal $T$-orbitally complete if and only if every Cauchy sequence $\left\{x_{n}\right\}$ is ordered proximal Picard sequence starting with some $x_{0} \in A_{0}$ converges to an element in the set $A_{0}$.

Lemma 5. Let $T: A \rightarrow B$ be continuous, proximally monotone and $\alpha-$ Pataproximal admissible contraction of type-II mapping with $A_{0} \neq \varnothing$ and $T\left(A_{0}\right) \subseteq B_{0}$. Suppose that each pair of elements in partially ordered complete metric spaces $(X, d, \preceq)$ has a lower and upper bound. Then $A_{0}$ is proximal $T$-orbitally complete provided that $T$ is one to one on $A_{0}$ also there exist a function $\alpha: A \times A \rightarrow[0, \infty)$ such that $(x, y) \in \Delta$ and $\alpha(x, y) \geq 1$ for all $x, y \in A$. 
Proof. Consider a function $\alpha: A \times A \rightarrow[0, \infty)$ such that

$$
\alpha(x, y)=\left\{\begin{array}{l}
1 \text { if }(x, y) \in \Delta, \\
0 \text { otherwise }
\end{array}\right.
$$

Let $x_{0}$ be a given point in $A_{0}$. Suppose that $\left\{x_{n}\right\}$ is an ordered proximal Picard sequence starting with $x_{0}$. As $(X, d, \preceq)$ is complete and $A$ is closed, there exist some $x^{*}$ in $A$ such that $\lim _{n \rightarrow \infty} d\left(x_{n}, x^{*}\right)=0$. As $\left\{x_{n}\right\}$ is a ordered proximal Picard sequence starting with $x_{0}$, we have

$$
d\left(x_{n}, T x_{n-1}\right)=d(A, B) \text { and } d\left(x_{n+1}, T x_{n}\right)=d(A, B)
$$

with $\left(x_{n-1}, x_{n}\right)$ belong to the set of all ordered proximal Picard sequence starting with $x_{0}$, for all $n \in \mathbb{N}$. Since $T$ is an $\alpha$ - Pata-proximal admissible contraction of type-II and function $\alpha$ defined in (4.2) which agrees with the $\alpha$-proximal admissible function defined on $A \times A$, rest of proof follows from the arguments on the same lines as in Theorem (3).

Theorem 3. Let $T: A \rightarrow B$ be a continuous, proximally monotone and $\alpha-$ Pataproximal admissible contraction of type-II with $A_{0} \neq \phi$ and $T\left(A_{0}\right) \subseteq B_{0}$. Suppose that each pair of elements in partially ordered complete metric spaces $(X, d, \preceq)$ has a lower and upper bound. If $B$ is approximately compact with respect to $A$, then $T$ has a unique best proximity point $x^{*}$ in $A_{0}$ for all $(x, y) \in \Delta$ such that $\alpha(x, y) \geq 1$ for all $x, y \in A$.

Proof. Let $x_{0}$ be a given point in $A_{0}$. From Lemma (4), the set of ordered proximal Picard sequence starting with $x_{0}$ is nonempty and $\left\{x_{n}\right\}$ belong to the set of ordered proximal Picard sequence starting with $x_{0}$ in $A_{0}$. We can obtain a sequence $\left\{x_{n}\right\}$ in $A_{0}$ such that it satisfies

$$
d\left(x_{n}, T x_{n-1}\right)=d(A, B), d\left(x_{n+1}, T x_{n}\right)=d(A, B)
$$

for all $n \in \mathbb{N}$. Define a function $\alpha: A \times A \rightarrow[0, \infty)$, which satisfies (4.2). Also, function $\alpha$ agrees with the $\alpha$-proximal admissible function defined on $A \times A$. Since $T$ is an $\alpha$ - Pata-proximal admissible contraction of type-II. Following arguments similar to those in the proof of Lemma (3) and Theorem (1), we can show the existence and uniqueness of best proximity point in partially ordered metric spaces.

Theorem 4. Let $g: A \rightarrow A$ be an expansive mapping and $T: A \rightarrow B$ be an $\alpha-$ proximal admissible with $A_{0} \neq \phi, T\left(A_{0}\right) \subseteq B_{0}$ and $A_{0} \subseteq g\left(A_{0}\right)$ for any $t>0$. If $B$ is approximately compact with respect to $A$ and the pair $(g, T)$ is generalized $\alpha-P a t a-$ proximal contraction. Then the pair $(g, T)$ has a unique optimal coincidence point $x^{*}$ in $A_{0}$ provided that $\alpha(x, y) \geq 1$ for all $x, y \in A$.

Proof. By Lemma (4), the set of ordered proximal Picard sequence starting with $x_{0}$ is nonempty. Let $x_{0}$ be a given point in $A_{0}$. As $T\left(A_{0}\right) \subseteq B_{0}$ and $A_{0} \subseteq g\left(A_{0}\right)$, we can choose an element $x_{1} \in A_{0}$ such that $d\left(g x_{1}, T x_{0}\right)=d(A, B)$, where $\left(x_{0}, x_{1}\right) \in \Delta$. Also, 
$T x_{1} \in T\left(A_{0}\right) \subseteq B_{0}$, and $A_{0} \subseteq g\left(A_{0}\right)$, it follows that there exist an element $x_{2} \in A_{0}$ such that $d\left(g x_{2}, T x_{1}\right)=d(A, B)$, since $T$ is ordered, where $\left(g x_{1}, g x_{2}\right) \in \Delta$. Continuing this way, we can obtain a sequence $\left\{g x_{n}\right\}$ in $A_{0}$ such that it satisfies

$$
d\left(g x_{n}, T x_{n-1}\right)=d(A, B) \text { and } d\left(g x_{n+1}, T x_{n}\right)=d(A, B),
$$

where $\left(x_{n-1}, x_{n}\right) \in \Delta$. Also, define a function $\alpha: A \times A \rightarrow[0, \infty)$ by

$$
\alpha(x, y)=\left\{\begin{array}{l}
1 \text { if }(x, y) \in \Delta \\
0 \text { otherwise }
\end{array}\right.
$$

Which agrees with $\alpha$-proximal admissible function. Following the same lines of the proof of Theorem (2), we have the result.

Corollary 2. If $T: A \rightarrow B$ is an $\alpha-$ Pata-proximal admissible contraction of typeI with $A_{0} \neq \phi$ and $T\left(A_{0}\right) \subseteq B_{0}$ for any $t>0$. Then $T$ has a unique best proximity point $x^{*}$ in $A_{0}$ provided that $B$ is approximately compact with respect to $A$.

Example 4. Suppose that $X=[0,1] \times \mathbb{R}$ is an orderded metric space $(X, d, \preceq)$, where $d(x, y)=|x-y|$ and $x \preceq y$ is defined as $x \leq y$. If $A=\{(0, x): x \in \mathbb{R}\}$ and $B=\{(1, y): y \leq 0, y \in \mathbb{R}\}$. Note that

$$
d(A, B)=1, A_{0}=\{(0,0)\} \text { and } B_{0}=\{(1,0)\} .
$$

Define $T: A \rightarrow B$ as:

$$
T(0, x)=(1,2 x) .
$$

Obviously, $T\left(A_{0}\right)=B_{0}$. Note that the points $u=\left(0, x_{1}\right), v=\left(0, x_{2}\right), x=\left(0, y_{1}\right)$ and $y=\left(0, y_{2}\right)$ in $A$ satisfy $d(u, T x)=d(A, B)$ and $d(v, T y)=d(A, B)$, if $x_{1}=2 y_{1}$ and $x_{2}=2 y_{2}$. Also, mapping $T$ satisfies the condition of $\alpha$ - Pata-proximal admissible contraction of type-I, where $\psi(t)=\sqrt{t}$. Thus all the conditions of the Corollary (2) are satisfied. Moreover, $(0,0)$ is the only best proximity point of $T$ in $A_{0}$.

Corollary 3. Let $g: A \rightarrow A$ be a isometric mapping and $T: A \rightarrow B$ with $A_{0} \neq \phi$, $T\left(A_{0}\right) \subseteq B_{0}$ and $A_{0} \subseteq g\left(A_{0}\right)$ for any $t>0$. If $B$ is approximately compact with respect to $A$ and the pair $(g, T)$ is generalized $\alpha$-Pata-proximal contraction. Then the pair $(g, T)$ has a unique optimal coincidence point $x^{*}$ in $A_{0}$.

\section{APPLICATION}

As an application of our results, we prove some new fixed point theorems as follows.

We start with the following fixed point theorem:

Theorem 5. Let $(X, d)$ be a complete ordered metric space, and $\alpha: A \times A \rightarrow \mathbb{R}$. Let $T: X \rightarrow X$ be a continuous and $\alpha$-admissible mapping satisfying

i): $\alpha(x, y) d(T x, T y) \leq \frac{(1-\varepsilon)}{2} d(x, y)+\Lambda \varepsilon^{\lambda} \psi(\varepsilon)[1+\|x\|+\|y\|+\|v\|]^{\mu}$.

ii): There exist $x_{0} \in X$ such that $\alpha\left(x_{0}, T x_{0}\right) \geq 1$. 
Then $T$ has a fixed point $x^{*} \in X$ and $\left\{T^{n} x_{0}\right\}$ converges to $x^{*}$.

Proof. Let $A=B=X$. We prove that $T$ is an $\alpha$ - Pata-proximal admissible contraction of type-I. Let $x, y, u, v \in X$, satisfy the following conditions:

$$
\left\{\begin{array}{l}
(x, y) \in \Delta, \\
d(u, T x)=d(A, B), \\
d(v, T y)=d(A, B) .
\end{array}\right.
$$

Since $X$ is complete ordered metric space, then $(T x, T y) \in \Delta$, Since $d(A, B)=0$, so we have $u=T x$ and $v=T y$, hence $(u, v) \in \Delta$. Since $T$ satisfies condition (i), we have

$$
\begin{aligned}
\alpha(x, y) d(u, v)=\alpha(x, y) d(T x, T y) & \\
& \leq \frac{(1-\varepsilon)}{2} d(x, y)+\Lambda \varepsilon^{\lambda} \psi(\varepsilon)[1+\|x\|+\|y\|+\|v\|]^{\mu},
\end{aligned}
$$

which implies that

$$
\alpha(x, y) d(u, v) \leq \frac{(1-\varepsilon)}{2} d(x, y)+\Lambda \varepsilon^{\lambda} \psi(\varepsilon)[1+\|x\|+\|y\|+\|v\|]^{\mu},
$$

and hence $T$ is an $\alpha$ - Pata-proximal admissible contraction of type-I. Let

$$
\left\{\begin{array}{l}
\alpha(x, y) \geq 1, \\
d(u, T x)=d(A, B), \\
d(v, T y)=d(A, B) .
\end{array}\right.
$$

Since $T$ is an $\alpha$-ordered admissible mapping. Applying condition (ii), there exists $x_{0} \in X$ such that $\alpha\left(x_{0}, T x_{0}\right) \geq 1$. If we choose $x_{1}=T x_{0}$, then we obtain that

$$
\alpha\left(x_{0}, x_{1}\right) \geq 1 \text { and } d(A, B)=d\left(x_{1}, T x_{0}\right)=d\left(T x_{0}, T x_{0}\right) .
$$

Set $B$ is approximately compact with respect to $A$, the conditions of Corollary (1) are satisfied, so there exists $x^{*} \in X$ such that $d\left(x^{*}, T x^{*}\right)=0=d(A, B)$, which implies that $T x^{*}=x^{*}$.

\section{CONCLUSION}

In this paper, we defined $\alpha$ - Pata-proximal admissible contraction of type-I and type-II in the framework of a complete metric space. Further, we obtained the best proximity point and optimal coincidence point results for such mappings. We proved the results which extends and generalize the existing results in $[7,8,10]$. In case, fixed points of mappings introduced in this paper do not exists, the results helps to obtain the approximate fixed points. To elaborate these concepts and support the results, some examples are presented.

Further, we developed some approximation results in complete ordered metric space which shows the validity of obtained results in ordered structure. Under some restriction, specially for self mapping, we obtained a fixed point result as an application of our result. 


\section{REFERENCES}

[1] S. Balasubramanian, "A Pata-type fixed point theorem." Math Sci, vol. 8, pp. 65-69, 2014.

[2] M. Chakraborty and S. K. Samanta, "A fixed point theorem for Kannan-type maps in metric spaces." IJMAA, vol. 6, no. 4, pp. 157-161, 2018.

[3] C. Di Bari, T. Suzuki, and C. Vetro, "Best proximity points for cyclic Meir-Keeler contractions." Nonlinear Anal., vol. 69, pp. 3790-3794, 2008, doi: https://doi.org/10.1007/s40096-014-0127-4.

[4] A. A. Eldred and P. Veeramani, "Existence and convergence of best proximity points." J. Math. Anal. Appl., vol. 323, pp. 1001-1006, 2006, doi: 10.1016/j.jmaa.2005.10.081.

[5] K. Fan, "Extensions of two fixed point Theorems of F. E. Browder." Math. Z., vol. 112, pp. 234240, 1969, doi: https://doi.org/10.1007/BF01110225.

[6] M. Jleli and B. Samet, "An optimization problem involving proximal contraction mappings." Fixed Point Theory and Appl., vol. 141, 2014, doi: https://doi.org/10.1186/1687-1812-2014-141.

[7] Z. Kadelburg and S. Radenović, "A note on Pata-type cyclic contractions." Sarajevo J. of Math., vol. 24, no. 2, pp. 235-245, 2015, doi: 10.5644/SJM.11.2.09.

[8] Z. Kadelburg and S. Radenović, "Fixed point theorem under Pata-type conditions in metric spaces." J. of Egyptian Math. Soc., vol. 24, pp. 77-82, 2016, doi: http://dx.doi.org/10.1016/j.joems.2014.09.001.

[9] E. A. Karapinar and I. M. Erhan, "Best proximity point on different type contractions." Appl. Math. Inf. Sci., vol. 5, no. 3, pp. 558-569, 2011.

[10] V. Pata, "A fixed point theorem in metric spaces." J. Fixed Point Theory Appl., vol. 10, pp. 299305, 2011, doi: https://doi.org/10.1007/s11784-011-0060-1.

[11] S. Sadiq Basha, "Best proximity point theorems on partially ordered sets." Optim. Lett., 2013, doi: https://doi.org/10.1007/s11590-012-0489-1.

[12] N. Shahzad, S. Sadiq Basha, and R. Jeyaraj, "Common best proximity points: global optimal solutions.” J. Optim. Theory Appl., vol. 148, pp. 69-78, 2011, doi: https://doi.org/10.1007/s10957010-9745-7.

[13] S. Wang, B. Li, Z. Gao, and K. Iseki, "Some fixed point theorems on expansion mappings." Math. Jpn. , vol. 29, pp. 631-636, 1984.

Authors' addresses

N. Saleem

Nonlinear Analysis Research Group, Ton Duc Thang University, Ho Chi Minh City, Vietnam Department of Mathematics and Statistics, Ton Duc Thang University, Ho Chi Minh City, Vietnam

E-mail address: naeem. saleem@tdtu.edu.vn

\section{Abbas}

Government College University, Department of Mathematics, Lahore, Pakistan.

China Medical University, Taichung 404, Taiwan

E-mail address: abbas.mujahidegmail.com

B. Bin-Mohsin

Department of Mathematics, College of Science, King Saud University, Riyadh 11451, Saudi Arabia

E-mail address: balmohsen@ksu.edu.sa

S. Radenović

Department of Mathematics, College of Science, King Saud University, Riyadh 11451, Saudi Arabia

E-mail address: radens@beotel. net 